\title{
Disc signatures in a new population of low mass YSOs in $\rho$ Ophiuchi
}

\author{
Catarina Alves de Oliveira ${ }^{1}$ and Mark Casali ${ }^{1}$ \\ ${ }^{1}$ ESO, Karl-Schwarzschild-Str. 2, \\ 85748 Garching bei München, Germany \\ email: coliveir@eso.org
}

\begin{abstract}
The deepest near-IR variability survey of the $\rho$ Ophiuchi cluster with the WFCAM/UKIRT has been used to uncover a new population of young low mass objects. Evidence for the existence of discs around the new objects has been found when combining data with IRAC/Spitzer observations. A new insight on the frequency and dynamics of discs around low mass YSOs is given, essential for understanding the origin and evolution of circumstellar discs and ultimately the environment for planet formation.
\end{abstract}

Keywords. Stars: pre-main-sequence,activity

\section{Introduction}

Variability is a common characteristic of pre-main-sequence stars (Joy 1942) and a useful tool for uncovering new cluster members in star formation regions. Young stellar objects (YSOs) are known to be variable in the near-IR, especially suitable for studying the circumstellar environment (e.g, Carpenter et al. 2001). With a large field of view ( $0.8 \mathrm{deg}^{2}$ in four exposures), the WFCAM IR imager (Casali et al. 2007) on the UK Infrared Telescope has made possible deep variability studies of nearby star forming regions which extend over degree scales. The results of such a study are presented, with multi-epoch $H$ and $K$ observations of the $\rho$ Ophiuchi cluster used to search for variability and uncover new pre-main-sequence stars (Alves de Oliveira \& Casali 2008). Combining recently released IRAC/Spitzer data from the $\mathrm{c} 2 \mathrm{~d}$ point-source catalogues of the third data delivery (Evans et al. 2005), the list of variable objects is searched for IR-excesses characteristic of stars surrounded by an accretion disc.

\section{Variable stars in $\rho$ Ophiuchi}

The reduced chi-square $\chi^{2}\left(\chi_{\nu}^{2}\right)$ of the magnitudes together with cross-correlation indexes are used to detect variability; 182 variable stars are found, 53 are previously known members of the cluster and the others are named candidate members. The colours and magnitudes of the variable stars are an important clue in investigating the youth and masses of these objects. The colour-magnitude diagram, $K$ vs. $H-K$, shown in Fig. 1 , displays the variable members of $\rho$ Ophiuchi (open triangles) and the candidate members (filled circles), overlaid on the distribution of all other detected sources, expected to be in its majority field stars. The models indicate that the brighter candidates are above the hydrogen burning limit, but the faint sources would extend to very low masses. 


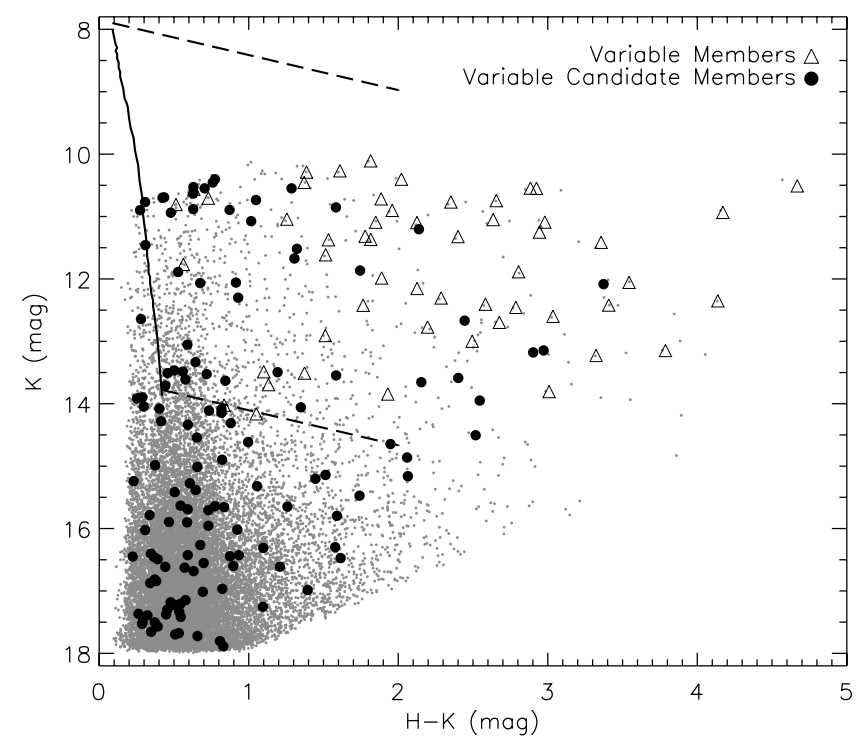

Figure 1. Colour-magnitude diagram for variable members and variable candidate members of $\rho$ Ophiuchi, overlaid on the distribution of all other detected sources. The locus for the $5 \mathrm{Myr}$ pre-main sequence down to $20 \mathrm{M}_{J u p}$ (Baraffe et al. 1998) is shown along with reddening vectors (Rieke \& Lebofsky 1985).

\section{Disc signatures}

Young stars show infrared emission which originates from dusty envelopes and circumstellar discs surrounding the central object. Lada \& Wilking (1984), based on the level of long wavelength excess with respect to a stellar photosphere emission, identified three different classes which define an IR or SED classification scheme: Class I, low mass protostars surrounded by an infalling envelope with large IR excess; Class II, young stars with accretion discs and a moderate IR excess (as classical T Tauri stars, CTTSs); and Class III, stars which no longer accrete matter from a circumstellar disc and show no IR excess (as weak-line T Tauri stars, WTTSs). The IRAC data from Spitzer allows the study of these objects in the mid-IR, where the excess contribution from discs and envelopes is predominant. The IRAC colour-colour diagram ([3.6]-[4.5] vs. [5.8]-[8.0]) was presented as a tool to separate young stars of different classes (Allen et al. 2004).

The Ophiuchus molecular cloud has been mapped with IRAC in the 3.6, 4.5, 5.8 and $8.0 \mu \mathrm{m}$ bands, over a region of $8.0 \mathrm{deg}^{2}$, which encompasses the WFCAM field. Figure 2 shows the IRAC colour-colour diagram for all variable objects with detections in the four bands, where the top diagram displays $\rho$ Ophiuchi members and the bottom diagram the candidate members. The objects are displayed accordingly to their class, i.e., Class I (open squares), Class II (filled circles), and Class III (open triangles). The extinction vector is from Flaherty et al. (2007). For comparison, a sample of objects has been chosen from a region of the sky away from the central cloud with little extinction which should mainly contain field stars. The objects tend to cluster around three main regions of the diagram. Centred in the origin, $[3.6]-[4.5],[5.8]-[8.0]=(0,0)$, are sources which have colours consistent with stellar photospheres and have no intrinsic IR excess. These can be foreground and background stars, but also Class III stars which do not have significant circumstellar dust. Another preferred region for objects in the diagram is located within the box defined by Allen et al. (2004) which represents the colours expected from models of discs around young, low-mass stars. In fact, many of the Class II objects classified by 


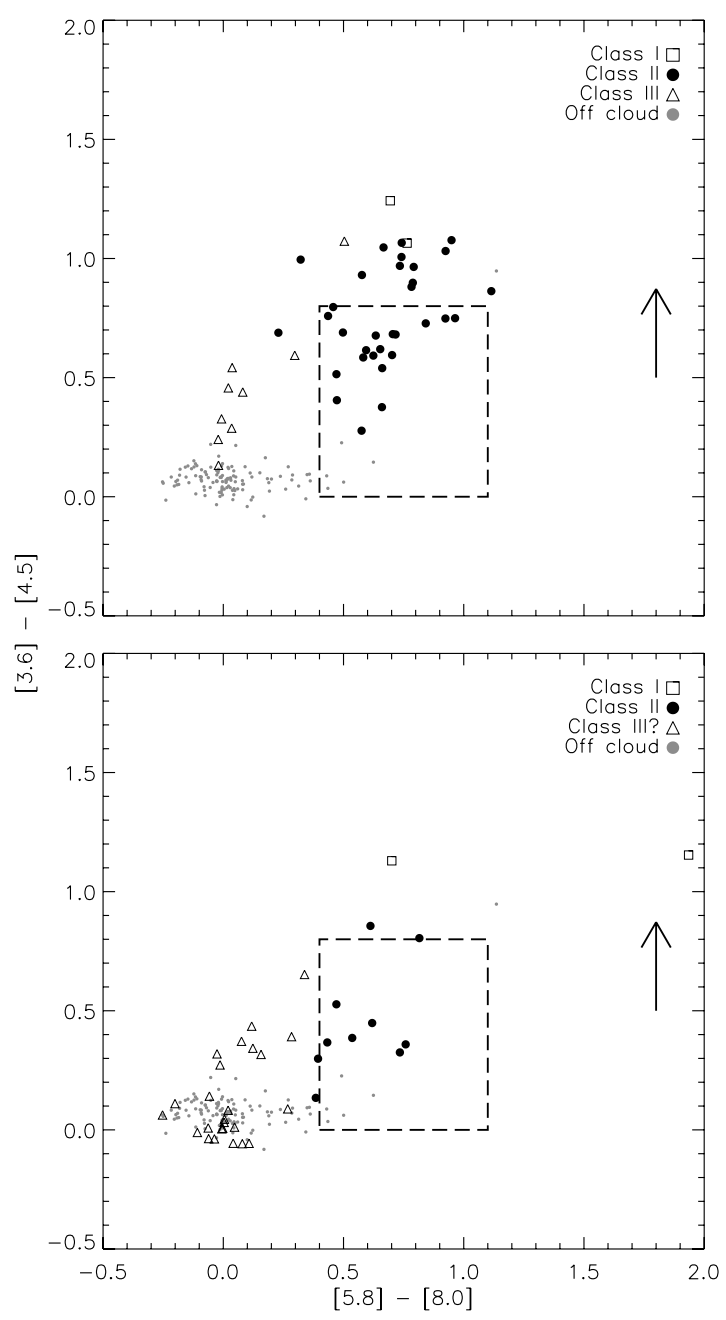

Figure 2. IRAC colour-colour diagram for variable members (top panel) and candidate members (bottom panel). Arrow represents the $\mathrm{A}_{k}=4$ extinction vector for $\rho$ Oph (Flaherty et al. 2007).

Bontemps et al. (2001) lie within that range. However, some sources previously identified as Class II do not fall within the predicted limits. These objects have colours inconsistent with Class II sources (higher [3.6]-[4.5] colours than Class II but lower [5.8]-[8.0] colours than Class I) and were previously classified as candidate flat spectrum objects. Their location in this diagram does not confirm them as transition objects between Class I and II (Bontemps et al. 2001) since they can be explained as reddened Class II sources. Finally, from models of infalling envelops, Allen et al. (2004) predict the colours of Class I sources to have $([3.6]-[4.5])>0.8$ and/or $([5.8]-[8.0])>1.1$, which agrees well with the two Class I objects identified with ISOCAM (Bontemps et al. 2001). The same criteria can be applied to classify the candidate member variables on the bottom panel of the diagram. Combining WFCAM near-IR observations with IRAC/Spitzer mid-IR data, it is possible to classify 12 of the candidate members of $\rho$ Ophiuchi as Class I/II, and 25 into possible Class III. Furthermore, the SED classes from the literature for the variable members are confirmed, with the exception of few objects. 


\section{Conclusions}

Using near-IR variability, 129 new candidate members of Ophiuchus are uncovered. Combined with IRAC/Spitzer data, a subsample of 10 candidate members is classified as Class II objects, believed to be YSOs with accretion discs. This study is an important contribution for obtaining a complete census of stars surrounded by circumstellar disks in nearby star forming regions. A new coeval sample of YSOs spanning a wide range of masses is presented, ideal to study the early onset of planet formation.

\section{Acknowledgements}

We thank the UKIRT observatory staff and WFCAM Science Archive team for acquiring and pipeline processing the observations used for this project. C. A. O. acknowledges partial support from a Marie Curie Fellowship for Early Research Training. This work is based in part on observations made with the Spitzer Space Telescope, which is operated by the Jet Propulsion Laboratory, California Institute of Technology under a contract with NASA.

\section{References}

Allen, L. E., Calvet, N., D'Alessio, P., Merin, B., Hartmann, L., Megeath, S. T., Gutermuth, R. A., Muzerolle, J., Pipher, J. L., Myers, P. C., \& Fazio, G. G. 2004, ApJS, 154, 363

Alves de Oliveira, C. \& Casali, M. 2008, submitted to A\&A

Baraffe, I., Chabrier, G., Allard, F., \& Hauschildt, P. H. 1998, A\&A, 337, 403

Bontemps, S., André, P., Kaas, A. A., Nordh, L., Olofsson, G., Huldtgren, M., Abergel, A., Blommaert, J., Boulanger, F., Burgdorf, M., Cesarsky, C. J., Cesarsky, D., Copet, E., Davies, J., Falgarone, E., Lagache, G., Montmerle, T., Pérault, M., Persi, P., Prusti, T., Puget, J. L., \& Sibille, F. 2001, A\&3A, 372, 173

Carpenter, J. M., Hillenbrand, L. A., \& Skrutskie, M. F. 2001, ApJ, 121, 3160

Casali, M., Adamson, A.,Alves de Oliveira, C. Almaini, O., Burch, K., Chuter, T., Elliot, J., Folger, M., Foucaud, S., Hambly, N., Hastie, M. Henry, D., Hirst, P., Irwin, M., Ives, D., Lawrence, A., Laidlaw, K., Lee, D., Lewis, J., Lunney, D., McLay, S., Montgomery, D., Pickup, A., Read, M., Rees, N., Robson, I., Sekiguchi, K., Vick, A., Warren, S., \& Woodward, B. 2007, A\& $A, 467,777$

Evans, II, N. J., Allen, L. E., Blake, G. A., Boogert, A. C. A., Bourke, T., Harvey, P. M., Kessler, J. E., Koerner, D. W., Lee, C. W., Mundy, L. G., Myers, P. C., Padgett, D. L., Pontoppidan, K., Sargent, A. I., Stapelfeldt, K. R., van Dishoeck, E. F., Young, C. H., \& Young, K. E. 2005, Third Delivery of Data from the c2d Legacy Project: IRAC and MIPS(Pasadena, SSC)

Flaherty, K. M.,Pipher, J. L.,Megeath, S. T.,Winston, E. M., Gutermuth, R. A., Muzerolle, J., Allen, L. E., \& Fazio, G. G. 2007, ApJ, 663, 1069

Joy, A. H. 1942, PASP, 54, 35

Lada, C. J. \& Wilking, B. A. 1984, ApJ, 287, 610

Rieke, G. H. \& Lebofsky, M. J. 1985, ApJ, 288, 618 